\title{
Integrating Power line and Wireless for Intelligent and Opportunistic Networking
}

\author{
Soma Pandey \\ Assistant Professor \\ CMR Institute of Technology \\ \#132, AECS Layout, ITPL Road, \\ Bangalore - 560037
}

\author{
Vijay Pande \\ Professor \\ CMR Institute of Technology \\ \#132, AECS Layout, ITPL Road, \\ Bangalore - 560037
}

\author{
Govind Kadambi \\ Professor and Dean \\ MS Ramaiah School of Advanced \\ Studies, New BEL Road \\ Bangalore - 560054
}

\begin{abstract}
In this paper we try to present a network architecture which leverages the power line for Opportunistic networking. We try to provide the autonomous working by use of 802.11 wireless mesh networks. This architecture is aimed at providing better Quality of Service without any additional infrastructure. This integration gives the high bandwidth of wired network to WiFi. The architecture focuses on providing wired backhaul of power line to 802.11s WiFi network. Then the combined network is systematically simplified by graph theory simplification techniques to reduce the network size considerably thereby shortening the route path. It also achieves Quality of Service as each wired backhaul subsequently leads to Internet Gateway. Increasing the backhauls means increase in number of gateways. We devise an intelligent algorithm which can switch between gateways with change in traffic and other overloading conditions thereby bringing the bandwidth within the wireless network at par with its wired counterparts.
\end{abstract}

\section{Categories and Subject Descriptors}

C.2.1 [Wireless Communication]: Design of wireless LAN, IEEE $802.11 \mathrm{~s}$, combining wired and wireless networks, IEEE 802.11s networks, Wireless LAN

\section{General Terms}

Design

\section{Keywords}

WMN, IEEE802.11s, Planar graph, Internet Gateway Placement, Mesh Portal Point, Mesh Router, multihop wireless network.

\section{INTRODUCTION}

Powerline has a widespread reach and the strong infrastructure to cater to any scalable network. Even then it has been less researched upon for networking purposes. The major reasons are the EMC problems and poor modulation techniques. As of today most of these problems are resolved. With the evolution of robust techniques like OFDM with FHSS, DSSS etc. powerline communication has become as efficient and at par with its other counterparts like fiber, DSL /ADSL etc. In fact in some cases it has become even more preferable to these other physical layer infrastructures. Only drawback of powerline can be that it is a wired network. So even if it is opportunistic it is definitely not autonomous.

Wireless mesh networks on the other hand can provide autonomous working but at the cost of opportunity. Mesh access points can cater to only small ranges and areas. Thereafter they have to rely back on the wired backhaul.

In this paper we have addressed the problem of channel assignment in an entirely different way. We provide a wired backhaul support to the wireless network. Next we propose an artificially intelligent routing scheme which switches the mesh access points from purview of one gateway to another based on bandwidth availability on respective gateways. Along with this we have reduce the need of radio spectrum to "use only when necessary' concept. This not only simplifies the channel assignment problem but also has paves way for a simplified representation of the mesh.

In subsequent sections we first define the problems which are prevalent in wireless mesh networks and propose the "Network Contraction Algorithm".

\section{RELATED WORK}

It is already shown by Francis De Costa [8], Raniwala et. al. [7], [10], Arindam et. al. [11] and others that efficiency of WMN increases when changed from single radio to multi radio. Raniwala has further solved the channel assignment problem by proposing multiple NIC (Network Interface Card), Hyacinth architecture and load balancing algorithm. Overall most of the previous work remains focused on issues such as various channel utilization methods, interference management and better routing protocols. Providing a local backhaul for wireless mesh nodes has received no attention at all

\section{MOTIVATION}

Most other networks fail to synergize with each other due to two major factors

1. IP bottleneck [1] : As explained in [1] IP layer is a huge bottle neck to implement AOC. This is not only a 
severe limitation for switching at layers 2 and 3 but also creates overheads in pricing and bandwidth sharing calculations .

2. Widespread infrastructure availability: Most of the wired infrastructures are not available on 'as is where is' basis. This discourages the opportunistic approach. Hence only the wireless counterparts remain to be explored.

The point emphasized in this paper is that next generation devices already have the capabilities to communicate on $\mathrm{WiFi}$, Bluetooth, infrared and other multiple communication channels. Empowered with cognitive radio and software defined radios, the latest devices can switch over seamlessly across all radio spectrum ranges with versatility and ease. But the switching occurs manually depending on the type of application. For triple play to happen autonomously the device operating system should be intelligent enough to change the communication links from one medium to another. For opportunistic choices the system has to allow for multiple wireless links to coexist. This can be achieved by introducing the concept of small-small wired backhauls. Multiple backhauls can support a variety of different ISPs through different wireless standards. This can happen only with a wired infrastructure which keeps on popping up as and when required. The widespread availability of powerline makes it a suitable candidate for this arrangement.

\section{NETWORK MODEL AND ASSUMPTIONS}

We assume a WMN consisting of fixed wireless router nodes. These static nodes form a multi-hop ad hoc network. Each node in this multi channel WMN is equipped with multiple 802.11s compliant NIC, and a BPL interface.

Some of the mesh nodes are connected to their neighbors over the powerline interface. A mesh node communicates with its neighboring node using wireless if and only if there is no powerline link available between them. The mesh network can be of any topology. Our solution is not dependent on any particular type of mesh topology. We assume that the nodes run a mesh MAC layer which allows them to make a choice between powerline and wireless interfaces. We also assume that powerline is capable of working as PLC system supporting LAN/WAN mode.

\section{PROBLEM FORMULATION}

\subsection{Channel Assignment Problem}

As per Arindam K Das et al. [11] and Raniwala [7], [10] static channel assignment for an 802.11 mesh pertains to allocation of channels to mesh nodes with one or more radio interfaces such that

i. Maximize the number of simultaneous transmissions.

ii. Minimize the size of a co-channel interference set

iii. Assign the channels such that load is balanced i.e. prefer to assign dedicated radio channels over more traffic burdened links whereas lightly loaded links can be shared between the users.
Our Network contraction algorithm presented in next sections reduces the number of nodes communicating on wireless link thereby simplifying channel allocation algorithm.

\subsection{Computation Cost of Routing Path}

The routing algorithm requires the calculation of minimum path to reach gateway. Most popular is the minimum spanning tree (MST) algorithm. With the increase in size of the mesh and in the number of hops, spanning tree and shortest path calculation gets intensive. The "Network Contraction Algorithm" reduces the size of graph thereby reducing the cost of computing the MST.

\subsection{Performance Degradation over Multiple Hops Problem}

Bandwidth degradation in a Wireless mesh occurs at the rate of 1 $/ 2^{n}: n \rightarrow$ no. of hops [8]. The local backhaul frees the radio links between the nodes thereby conserving bandwidth. The "Network Contraction Algorithm" simplifies the network and reduces the number of hops required to reach the gateway.

\subsection{Seamless Handoff and Handover Problem}

Seamless handoff and handover is a challenging task to provide mobility to end users without being disconnected. This becomes difficult due to the channel assignment problem and constraints posed by the channel assignment algorithm. Further complications arise due to broadcast packets concerning mobility of the subscriber stations. Using the local backhaul, we can position routers such that the handoff mechanism is administrated locally. This minimizes the need for broadcasting location of end user. This is further discussed under section .

\section{LIST OF SYMBOLS}

$\begin{array}{lll}\text { WMN } & : & \text { Wireless Mesh Network } \\ \text { BPL } & : & \text { Broadband Power line } \\ \text { MST } & : & \text { Minimum Spanning Tree } \\ \mathrm{V}_{\mathrm{i}} & : & \text { The ith Vertex or the ith node } \\ \mathrm{E}_{\mathrm{i}} & : \text { Edge of graph showing connection between two nodes } \\ \mathrm{Ch}_{\mathrm{i}} & \text { :is the } \mathrm{i}^{\text {th }} \text { channel }\end{array}$

in the simplest mesh

Cell : A smallest cell area is area bounded by three distinct channels

$\mathrm{G}_{\mathrm{i}} \quad$ : Graph $\mathrm{i}$ constituting of distinct cell with reusable channel

$\mathrm{Gw}_{\mathrm{i}} \quad: \mathrm{i}^{\text {th }}$ Gateway for Broadband over Powerline Contracting : Collating two/three distinct nodes by wired medium (BPL in LAN mode)

\section{NETWORK CONTRACTION METHOD}

Consider the mesh network implementation in the last mile with dual link radio access nodes - one for the mesh and other for the 
mobility. From the practical stand point, these access nodes are pole top devices in the last mile

Each WMN node can have access to BPL as well as to the wireless mesh with help of dual radio link. Figure-1 is physical representation of the hybrid WiFi mesh network supported by Powerline backhaul. This is modeled as a Graph $G$ in the algorithm. The graph is divided into individual cell clusters $\mathrm{G}_{\mathrm{i}}$

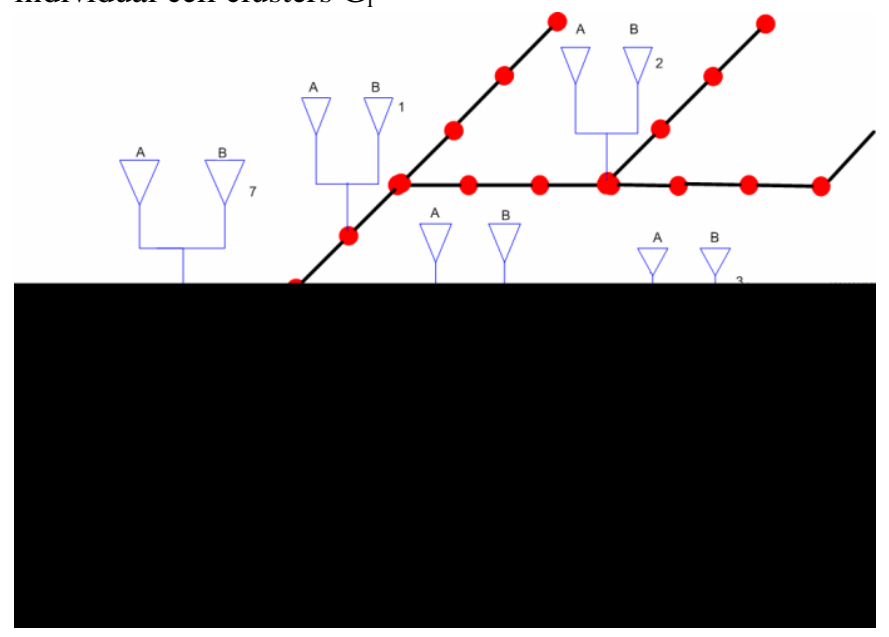

Figure 1: BPL access network with wireless mesh access points

using the Min-cut Algorithm [1]. One instance of this sub graph (cell cluster) is represented in figure 2. To explain the algorithm we consider a cell cluster of six nodes with each node connected to three neighbors. The network is modeled as a graph with six edges and seven nodes (Figure-2). For the sake of mobility requirements we use an undirected graph as each node has to do table management. This means that the span of the graph can be defined by the numbers of nodes. $V_{7} V_{4} V_{5}$ - forms a simple cell represented as $\mathrm{G}_{\mathrm{i}}$ in $\mathrm{G}$ (refer list of symbols). The spanning tree for mobility is shown in figure-6. [2], [3].So for simple mesh network with three access channel, we need to have, for mobility, spanning tree (figure 3 ), for mesh with 7 nodes. In the subsequent sections we explain this graph is simplified using the concept of backhauling and contraction. Later we will discuss how the channel assignment problem gets simplified

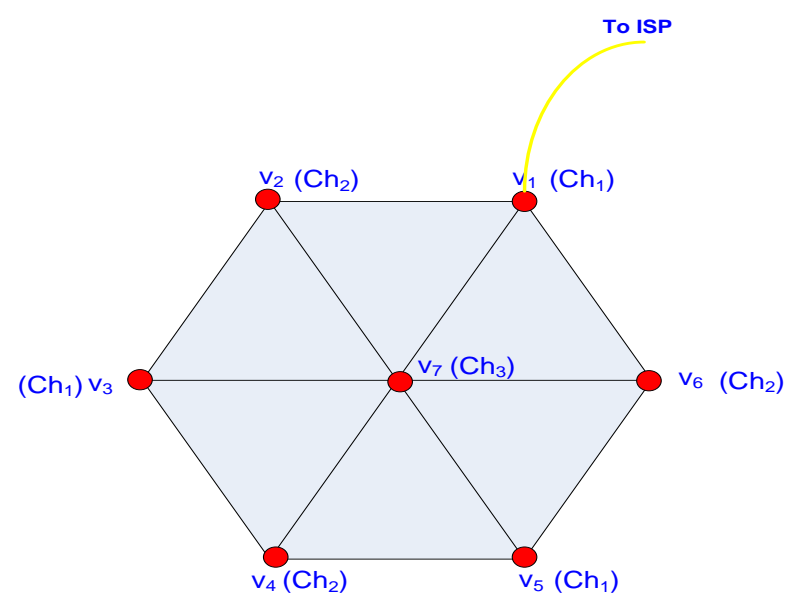

Figure 2. Undirected graph (G) with access channel Chi

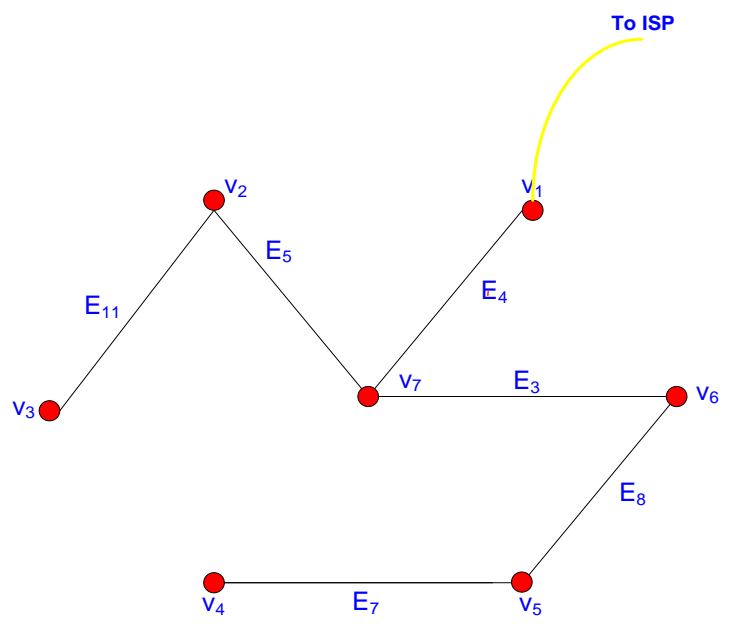

Figure 3: Spanning tree derived from graph of figure 2

Considering the assumptions listed under section (VI), about BPL access path, we add a BPL backhaul line to the topology depicted in figure 2. This is shown in figure 4 . The backhaul connects nodes $\mathrm{V}_{4}, \mathrm{~V}_{5}$ and $\mathrm{V}_{6}$. These three can be collated in to a single wireless node as three can communicate on the much higher bandwidth backhaul link Figure 4-1 shows the reduced graph 
Volume 1 - No. 25

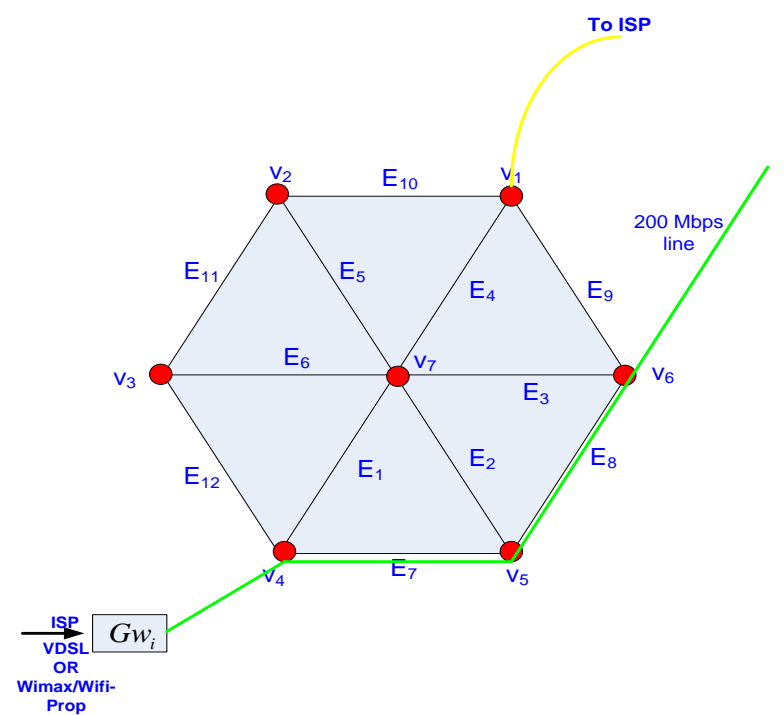

Figure 4: Hybrid access network with three vertices V4, V5 and V6 overlapping with power line

This contracted graph is reduced to a spanning tree represented in figure 4-2 [1].This network has not only improved the bandwidth but also has introduced additional handover points. Rebuilding of a mesh network from this spanning tree, results in lowering the hop cost.
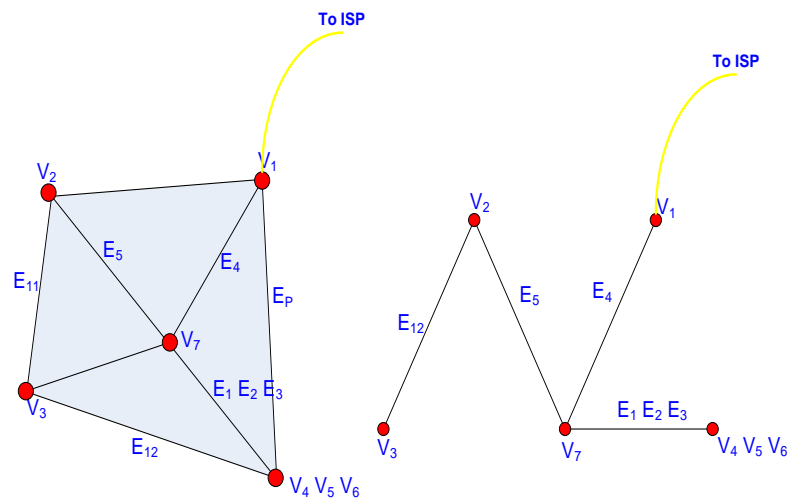

Fig 4-1 : Graph obtained tree

By contracting three nodes

V4, V5 and V6

As discussed earlier, once we contract $\mathrm{V}_{4}, \mathrm{~V}_{5}$ and $\mathrm{V}_{6}$ to one point, the links $\mathrm{E}_{1}, \mathrm{E}_{2} \mathrm{E}_{3} \mathrm{E}_{7}$ and $\mathrm{E}_{8}$ also get contracted leading into one link or usage of only one channel for the mesh. In a similar manner as explained, an intelligent algorithm contracts all adjacent channel pairs e.g. $\left(\mathrm{V}_{4}\right.$ and $\left.\mathrm{V}_{5}\right),\left(\mathrm{V}_{3}, \mathrm{~V}_{2}\right)$ and $\left(\mathrm{V}_{1}\right.$, $\mathrm{V}_{6}$ ) in figure 4 , then the total number of links get reduced to $50 \%$ of the original number.

This algorithm is repeated for each of the cell clusters. Some of the boundary nodes of the resulting sub-mesh networks will again be connected to the power-line back haul. This generates an opportunity for further collating these nodes across the sub-nets. This procedure is repeated recursively till the radio links of all the nodes connected by backhaul are contracted and a final graph remains with only those nodes which are communicating over wireless.

\section{NETWORK CONTRACTION ALGORITHM}

Notations

- Wireless mesh network with overlapping powerline be represented as Graph $\mathrm{G}$

- $\mathrm{G}$ represented by set of vertices $\mathrm{V}_{\mathrm{i}}$ and set of links $\mathrm{L}$

- $\mathrm{L}=\mathrm{L}_{1} \mathrm{U} \mathrm{L}_{2} \mathrm{U} \mathrm{L}_{3} \ldots \mathrm{U} \mathrm{L}_{\mathrm{N}}$

- $\mathrm{L}_{\mathrm{i}}$ set of all links incident on vertex $\mathrm{V}_{\mathrm{i}}$

$N \leftarrow$ total number of nodes/vertices

$L \leftarrow\{$ set of links/edges in $G\}$

$V \leftarrow\{$ set of vertices in $G\}$

$C \leftarrow$ no.of contracted nodes initialized to 0

procedure NetworkContraction $(N, L, G, V)$

\{

for $i=1$ to $\mathrm{Ndo}$

i

$$
\text { for } j=1 \text { to } \mathrm{Ndo}
$$

\{

If $\mathrm{V}_{\mathrm{i}}$ is adjacent to $\mathrm{V}_{\mathrm{j}}$ over powerline then

$\operatorname{Contract}\left(\mathrm{V}_{\mathrm{i}}, \mathrm{V}_{\mathrm{j}}, \mathrm{L}, \mathrm{N}, \mathrm{C}\right)$;

\}

\}

\}

procedure Contract $\left(V, \mathrm{~V}_{\mathrm{i}}, \mathrm{V}_{\mathrm{j}}, \mathrm{L}, \mathrm{N}\right)$

\{

$$
\begin{aligned}
& \mathrm{L}_{\mathrm{k}}=\mathrm{L}_{\mathrm{i}} \mathbf{U}_{\mathrm{j}} ; \\
& \mathrm{V}=\mathrm{V}-\left(\mathrm{V}_{\mathrm{i}}+\mathrm{V}_{\mathrm{j})}+\mathrm{V}_{\mathrm{k}} ;\right. \\
& \mathrm{L}=\mathrm{L}-\left(\mathrm{L}_{\mathrm{i}}+\mathrm{L}_{\mathrm{j}}\right)+\mathrm{L}_{\mathrm{k}} ; \\
& \mathrm{N}=\mathrm{N}-1 ;
\end{aligned}
$$$$
\mathrm{C}=\mathrm{C}+1
$$

Return (V,L, N,C);

\}

\section{IMPROVEMENT OVER CHANNEL BANDWIDTH}

Estimated Bandwidth gain for complete mesh cell

Total channels required $=\frac{N(N-1)}{2}$ 
Where N: no. of nodes

Let, $\mathrm{N}^{\prime}$ be the number of nodes in the mesh formed after adding the backhaul It is imperative that $\mathrm{N}^{\prime}<<\mathrm{N}$

Total bands required in the new network $=\mathrm{N}^{\prime}\left(\mathrm{N}^{\prime}-1\right) / 2$

For example if new network contains only $80 \%$ of the existing nodes communicating on wireless, the gain will be

$\frac{\frac{N(N-1)}{2}-\frac{0.8 N(0.8 N-1)}{2}}{\frac{N(N-1)}{2}}$

This gets reduced to

$\frac{0.36 N}{N-1}$

$\sim 36 \%$ for large $\mathrm{N}$,

\section{FUTURE WORK}

This work is being further extended to

- Intelligent algorithm to select the optimum set of nodes that need to be connected using local backhaul.

- Refine algorithm to incorporate uniformity in QoS and bandwidth across the mesh

- Redefining channel allocation problem on the reduced graphs with multiple gateways.

- Opening new opportunities for introduction of edge routers thereby allowing for multiple gateways to get associated with WiMAX mesh.

- Optimization and selection of backhaul gateways and positioning on the nodes for better network throughput.

- Load distribution amongst these gateways

- Performance optimization of medium independent handovers.

Outcome shall be reflected in our subsequent papers with field implementation results.

\section{CONCLUSION}

As various networking technologies mature in terms of bandwidth and quality of service, the focus will shift on seamless integration between these autonomous networks. In this paper we have presented a hybrid model whereby we can also leverage the wired broadband over powerline infrastructure for the autonomous wireless world. This way we improve upon the efficiency of the wireless and at the same time maintain the autonomous and opportunistic approach

\section{APPENDIX}

Elementary contraction operations

\section{Contraction of an edge:}

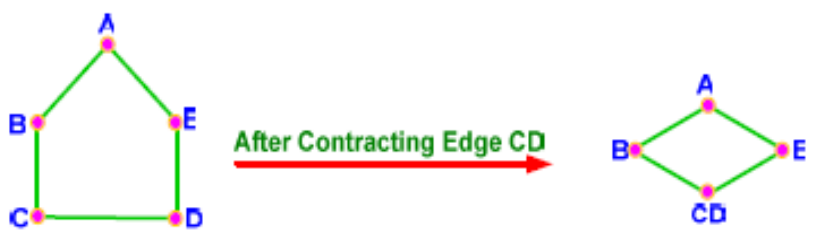

Figure 5 : Example of contracting edge CD

When contracting an edge, the edge $\mathrm{AB}$ is squeezed until the vertices $\mathrm{A}$ and $\mathrm{B}$ coincide and become one vertex, dragging the edges that had been attached at $\mathrm{A}$ and at $\mathrm{B}$ along, so that they now combine to contribute to the valence at the new combined vertex. The edge $\mathrm{AB}$ can now be thought of as being a loop at the combined vertex, but usually this loop is considered to be thrown away as part of the contraction process.[17]. A similar operation is vertex contraction, where we merge together two or more vertices, removing any edges between two of the vertices being contracted.

\section{Contraction of a path:}

Another related operation, sometimes called path contraction, is where we take a non-branching path of edges and contract it into a single edge (Figure 10). Edges to or from vertices along the path are either forbidden, arbitrarily connected to either endpoint of the new edge, or systematically connected to one endpoint.[13]
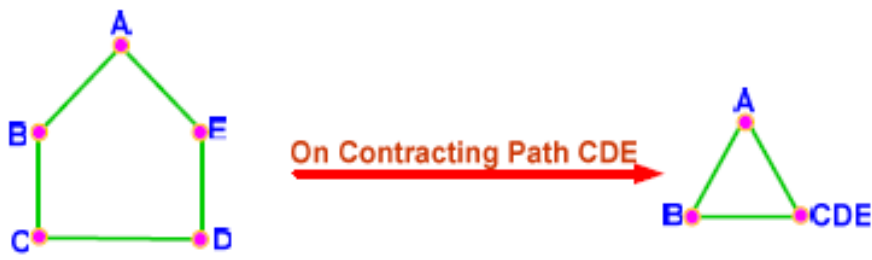

Graph G

Graph H

Figure 6 : Example of contracting path $\mathrm{CDE}$ and graph $\mathrm{G}$ to $\mathbf{H}$

\section{REFERENCES}

[1] Christophe Jelger, Christian Tschudin, Stefan Schmid, Guy Leduc, "Basic Abstractions for an Autonomic Network Architecture", AOC Workshop, Helsinki, June 2007.

[2] Oleksandr Grygorash, Yan Zhou and Zach Jorgensen, "Minimum Spanning Tree Based Clustering Algorithms," Proceedings of the IEEE International Conference on Tools with Artificial Intelligence (ICTAI'06), pp. 73-81, November 2006.

[3] Jun Han, Zhaohao Sun , Jinpeng Huai ,and Xian Li, “An Efficient Node Partitioning Algorithm for the Capacitated Minimum Spanning Tree Problem," IEEE/ACIS 
International Conference on Computer and Information Science (ICIS 2007), pp. 575-580, July 2007

[4] L.Lovasz, "On decompositions of graphs," Stud. Sci. Math. Hung., vol.1, pp. 237-238, 1966.

[5] P. Gupta and P.R. Kumar, "The capacity of wireless networks," IEEE Trans. Inform. Theory, vol. 46, no. 2, pp. 388-404, 2000.

[6] Fouad Tobagi, "Modelling and Performance analysis of multi-hop packet radio networks," Proceedings of the IEEE, vol. 75, no. 1, pp. 135-155

[7] Ashish Raniwala, Kartik Gopalan and Tzi cker Chiueh, “ Centralized channel assignment and routing algorithms for multi-channel wireless mesh networls," SIGMOBILE
Mobile computing Communications Review, Vol. 8, no. 2, pp. 50-65, 2004

[8] Mesh Dynamics; http://www.meshdynamics.com/performance-analysis.html

[9] H.Hrasnica, R. Lehnert, Powerline Communications in Telecommunication Access Area (Powerline Communications im TK-Zugangsbereich), VDE World Microtechnologies Congress - MICRO.tec 2000 - ETGFactagungund- Forum: Verteilungsnetze im liberalisierten Markt - Spectember 25-27, 2000 - Expo 2000, Hannover, Germany.

[10] Ashish Raniwala and Tzi cker Chiueh, "Architecture and algorithms for an IEEE 802.11-based multi-channel wireless mesh network," Proc. Of IEEE INFOCOM, 2005. 